\title{
SOME PROPERTIES OF MULTIPLICATION MODULES
}

\author{
H. A. Tavallaee ${ }^{1}$ and R. Mahtabi ${ }^{2}$ \\ ${ }^{1}$ Department of Mathematics, Tehran-North Branch, \\ Islamic Azad University, Tehran, Iran \\ tavallaee@iust.ac.ir \\ ${ }^{2}$ Faculty of sciences, University of Garmsar, \\ P.O.Box 318000062, Garmsar, Iran \\ r.mahtabi@gmail.com
}

\begin{abstract}
Let $M$ be an $R$-module. The module $M$ is called multiplication if for any submodule $N$ of $M$ we have $N=I M$, where $I$ is an ideal of $R$. In this paper we state some basic properties of submodules of these modules. Also, we study the relationship between the submodules of a multiplication $R$-module $M$ and ideals of ring $R$. Finally, by definition of semiprime submodule, we state some properties of radical submodules of multiplication modules.
\end{abstract}

Key words and Phrases: Multiplication module, prime submodule, primary submodule.

\begin{abstract}
Abstrak. Diberikan ring $R$ dan diketahui $M$ adalah $R$-modul. Modul $M$ disebut modul multiplikasi jika untuk setiap submodul $N$ di $M$ memenuhi sifat $N=I M$, untuk suatu ideal $I$ di $R$. Dalam paper ini diberikan beberapa sifat dasar submodulsubmodul dalam modul multiplikasi. Selain itu diberikan juga hubungan antara submodul di dalam modul multiplikasi $M$ atas $R$ dan ideal-ideal di dalam ring $R$. Dengan menggunakan definisi submodul semiprima, dihasilkan juga beberapa sifat submodule radikal dalam modul multiplikasi.
\end{abstract}

Kata kunci: Modul multiplikasi, submodul prima, submodul utama.

\section{INTRODUCTION}

In this paper all rings are commutative with identity and all modules over rings are unitary. Let $K$ and $N$ be submodules of an $R$-module $M$, we recall that $\left(N:_{R} K\right)=(N: K)=\{r \in R \mid r K \subseteq N\}$, which is an ideal of $R$. Let $N$ be a proper submodule of an $R$-module $M$, then $N$ is called a prime submodule of $M$,

2000 Mathematics Subject Classification: 13E05, 13E10, $13 \mathrm{C} 99$.

Received: 2 Aug 2016, revised: 11 June 2017, accepted: 11 June 2017. 
if for every $r \in R, x \in M ; r x \in N$ we have $x \in N$ or $r \in(N: M)$. In such a case $p=(N: M)$ is a prime ideal of $R$ and $N$ is said to be $p$-prime. The set of all prime submodules of $M$ is denoted by $\operatorname{Spec}(M)$ and for a submodule $N$ of $M$, $\operatorname{rad}(N)=\bigcap_{L \in \operatorname{Spec}(M), N \subseteq L} L$. If no prime submodule of $M$ contains $N$, we write $\operatorname{rad}(N)=M$. Also the set of all maximal submodules of $M$ is denoted by $\operatorname{Max}(M)$ and $\operatorname{Rad} M=\bigcap_{P \in \operatorname{Max}(M)} P$. Also we recall that if $I$ is an ideal of a ring $R$, then radical of $I$, i.e., $r(I)$ is defined as $\left\{r \in R \mid \exists k \in \mathbb{N} ; r^{k} \in I\right\}$. Now, let $\underline{a}$ be an ideal of a ring $R$ and $\underline{a}=\bigcap_{i=1}^{l} q_{i}$, where $r\left(q_{i}\right)=p_{i}$ is a normal primary decomposition of $\underline{a}$, then $\operatorname{ass}(\underline{a})=\left\{p_{1}, \ldots, p_{l}\right\}$.

An $R$-module $M$ is called a multiplication module if for every submodule $N$ of $M$ there exists an ideal $I$ of $R$ such that $N=I M$. It can be shown that $N=(N: M) M$.

\section{Definitions And Results}

Lemma 2.1. Let $R$ be a non-trivial ring and let $M$ be a multiplication $R$-module. Then $I M \neq M$ for any proper ideal $I$ of $R$.

Proof. Let $I$ be an arbitrary proper ideal of $R$, then there exists a maximal ideal $\underline{m}$ of $R$ such that $I \subseteq \underline{m}$. We remind that $R_{\underline{m}}=S^{-1} R$ and $M_{\underline{m}}=S^{-1} M$ are quotient ring of $R$ and quotient module respectively where $S=R-\underline{m}$.

We show that $\underline{m} M \neq M$. By [1, Lemma 2(i)], $M_{\underline{m}}$ is a multiplication $R_{\underline{m}}$-module and also by [3, Theorem 2.5], $\left(\underline{m} R_{\underline{m}}\right) M_{\underline{m}}$ is the only maximal submodule of $M_{\underline{m}}$. Thus $\left(\underline{m} R_{\underline{m}}\right) M_{\underline{m}}=(\underline{m} M)_{\underline{m}} \neq M_{\underline{m}}$ and hence $\left((\underline{m} M)_{\underline{m}}: M_{\underline{m}}\right)=\underline{m} R_{\underline{m}}$. Therefore $\underline{m} M \neq M$.

Now since $I M \subseteq \underline{m} M \neq M$, therefore $I M \neq M$ for every proper ideal $I$ of $R$.

Lemma 2.2. Let $M$ be a multiplication $R$-module, then $p M \in \operatorname{Spec}(M)$ and ( $p M$ : $M)=p$ for every $(0) \neq p \in \operatorname{Spec}(R)$.

Proof. Let $M$ be a multiplication $R$-module and let $(0) \neq p \in \operatorname{Spec}(R)$ be arbitrary. By Lemma 2.1, $p M \neq M$. We show that $(p M: M)=p$.

Let $r \in(p M: M)$ be arbitrary then $r M \subseteq p M$ and hence we have:

$$
(r M)_{p} \subseteq(p M)_{p} \Longrightarrow \frac{r}{1} M_{p} \subseteq(p M)_{p} \Longrightarrow \frac{r}{1} \in\left((p M)_{p}: M_{p}\right) .
$$

But by [1, Lemma 2(i)], $M_{p}$ is a multiplication $R_{p}$-module and by [3, Theorem 2.5], $(p M)_{p}$ is the only maximal submodule of $M_{p}$ and so $\left((p M)_{p}: M_{p}\right)=p R_{p}$. Thus $r \in p$ and hence $(p M: M)=p$.

Now by [2, Corollary 2], $p M$ is a primary submodule of $M$ and also by [5, Proposition 1], $p M \in \operatorname{Spec}(M)$. The proof is now completed. 
Corollary 2.3. Let $R$ be an arbitrary ring and let $M$ be a multiplication $R$-module. Then there exists a bijection between non-zero prime ideals of $R$ and non-zero prime submodules of $M$.

Proof. We show that for every $N \in \operatorname{Spec}(M), N=p M$ where $p \in \operatorname{Spec}(R)$. First, we show that, if $M$ is a multiplication $R$-module and $N$ is a submodule of $M$, then $N=(N: M) M$. Since $M$ is a multiplication $R$-module, hence there exists an ideal $I$ of $R$ such that $N=I M$. From this, we have $I \subseteq(N: M)$, and $N=I M \subseteq(N: M) M \subseteq N$, hence $N=(N: M) M$. Second, let $N \in \operatorname{Spec}(M)$. Since $M$ is a multiplication $R$-module, $N=(N: M) M$ and by [5, Proposition 1], $(N: M) \in \operatorname{Spec}(R)$.

Now we define $\psi: \operatorname{Spec}(M) \longrightarrow \operatorname{Spec}(R)$ by $\psi(p M)=(p M: M)$ for any non-zero prime ideal $p$ of $R$. Clearly $\psi$ is well defined and by Lemma $2.2, \psi$ is a bijection.

Proposition 2.4. Let $M$ be a faithful multiplication $R$-module. Then every proper direct summand of $M$ is prime. Hence $M$ is indecomposable.

Proof. By [3, Lemma 4.1], $M$ is torsion-free and by [5, Result 1], every direct summand of $M$ is a prime submodule.

Now we show that $M$ is indecomposable. If $M=M_{1} \oplus M_{2}$ where $M_{1}, M_{2} \neq 0$ then by the current form of the proposition, $M_{1}$ is a $p$-prime for some ideal $p$ of $R$. Thus $M_{1}=p M=p M_{1} \oplus p M_{2}$. Hence $p M_{2}=0$. Since $M$ is torsion-free and $M_{2} \neq 0$, we have $p=(0)$ and hence $M_{1}=0$, a contradiction.

Proposition 2.5. Let $M$ be a multiplication $R$-module. Then for every submodule $I M$ of $M$, if $I M \subseteq p M$ where $p \in \operatorname{Spec}(R)$, then $I \subseteq p$.

Proof. Let $I M \subseteq p M$ for $I \unlhd R$ and $p \in \operatorname{Spec}(R)$. Since $I \subseteq(I M: M) \subseteq(p M$ : $M)$, then by Lemma $2.2, I \subseteq p$.

Corollary 2.6. If $M$ is a faithful multiplication $R$-module then $M$ is finitely generated.

Proof. Since $M$ is a multiplication $R$-module, hence by Lemma $2.1, M \neq I M$ for every proper ideal of $I$ of $R$. Now, since $M$ is a faithful multiplication $R$-module, then by [3, Theorem 3.1 part (i), (iv)], $M$ is finitely generated.

Corollary 2.7. Let $M$ be a faithful multiplication $R$-module. Then for every ideal $I$ of $R,(I M: M)=I$.

Proof. Let $M$ is a faithful multiplication $R$-module, then by Corollary 2.6, $M$ is a finitely generated $R$-module. Now, let $(I M: M)=q$ where $I$ and $q$ be ideals of $R$. Since $(I M: M)=q, q M \subseteq I M$ and by [3, Theorem 3.1 part (ii)], $q \subseteq I$. Now, since $I \subseteq(I M: M)=q$, therefore $I=(I M: M)$.

Lemma 2.8. If $M$ is a faithful multiplication $R$-module. Then there exists a bijection between ideals of $R$ and submodules of $M$.

Proof. Since $M$ is a multiplication $R$-module, hence for every submodule $N$ of $M$ there exists an ideal $I$ of $R$ such that $N=I M$ and by Corollary 2.7, $(N: M)=$ 
$(I M: M)=I$. Now we define $\psi: M \longrightarrow R$ by $\psi(I M)=(I M: M)$ for any ideal $I$ of $R$. Obviously $\psi$ is well defined and also $\psi$ is an epimorphism. Now let $N_{1}, N_{2}$ be submodules of $M$, then there exist $I_{1}, I_{2} \unlhd R$ such that $N_{1}=I_{1} M$ and $N_{2}=I_{2} M$. If $\psi\left(N_{1}\right)=\psi\left(N_{2}\right)$ then $\left(I_{1} M: M\right)=\left(I_{2} M: M\right)$ and by Corollary 2.7, $I_{1}=I_{2}$. Therefore $\psi$ is a bijection.

Corollary 2.9. Let $M$ be a Noetherian multiplication $R$-module. Then $R$ satisfies the ascending chain condition on prime ideals.

Proof. Let $p_{1} \subseteq p_{2} \subseteq p_{3} \subseteq \ldots$ be an ascending chain of prime ideals of $R$. Then $p_{1} M \subseteq p_{2} M \subseteq p_{3} M \subseteq \ldots$ But, $M$ is a Noetherian $R$-module, hence there exists submodule (by [3, Theorem 2.5 part (i)], specially a maximal submodule ) $N$ of $M$ such that $p_{1} M \subseteq p_{2} M \subseteq p_{3} M \subseteq \ldots \subseteq N$. But $M$ is a multiplication $R$-module, hence by [3, Theorem 2.5 part (ii)], there exists a maximal ideal $\underline{m}$ of $R$ such that $N=\underline{m} M$. So we have $p_{1} M \subseteq p_{2} M \subseteq p_{3} M \subseteq \ldots \subseteq \underline{m} M$ and hence $\left(p_{1} M: M\right) \subseteq\left(p_{2} M: M\right) \subseteq\left(p_{3} M: M\right) \subseteq \ldots \subseteq(\underline{m} M: M)$. Now by Lemma 2.2 , $p_{1} \subseteq p_{2} \subseteq p_{3} \subseteq \ldots \subseteq \underline{m}$. The proof is now completed.

Corollary 2.10. Let $R$ be an arbitrary ring and let $M$ be a multiplication $R$ module. Then $\operatorname{Ann}_{R}(M) \subseteq p$ for each $(0) \neq p \in \operatorname{Spec}(R)$.

Proof. By the Lemma 2.2, $p M \in \operatorname{Spec}(M)$ for every $(0) \neq p \in \operatorname{Spec}(R)$. Therefore by [3, Corollary 2.11 part (i), (iii)], $A n n_{R}(M) \subseteq p$.

We recall that in the following lemma $J(R)$ and $\underline{n}_{R}$ denote the Jacobson radical and nilradical of $R$, respectively.

Lemma 2.11. Let $R$ be a ring and $M$ a multiplication $R$-module. Then $\bigcap_{\lambda \in \Lambda}\left(p_{\lambda} M\right)=$ $\left(\bigcap_{\lambda \in \Lambda} p_{\lambda}\right) M$ for any non-empty collection of non-zero prime ideals $p_{\lambda}(\lambda \in \Lambda)$ of $R$. Also if $R$ is a ring which is not an integral domain then $\bigcap_{0 \neq P \in \operatorname{Spec}(M)} P=\underline{n}_{R} M$ and $\operatorname{Rad} M=J(R) M$.

Proof. Let $M$ be a multiplication $R$-module and let $p_{\lambda}(\lambda \in \Lambda)$ be any non-empty collection of non-zero prime ideals of $R$. By [3, Corollary 1.7], $\bigcap_{\lambda \in \Lambda}\left(p_{\lambda} M\right)=$ $\left(\bigcap_{\lambda \in \Lambda}\left[p_{\lambda}+A n n_{R}(M)\right]\right) M$. But by Corollary $2.10, \bigcap_{\lambda \in \Lambda}\left(p_{\lambda} M\right)=\left(\bigcap_{\lambda \in \Lambda} p_{\lambda}\right) M$.

By Lemma $2.2, \bigcap_{0 \neq P \in \operatorname{Spec}(M)} P=\bigcap_{(0) \neq p \in \operatorname{Spec}(R)}(p M)$ and also by above we have $\bigcap_{(0) \neq p \in \operatorname{Spec}(R)}(p M)=\underline{n}_{R} M$. So $\bigcap_{0 \neq P \in \operatorname{Spec}(M)} P=\underline{n}_{R} M$. Also by Lemma 2.2, $\operatorname{Rad} M=\bigcap_{\underline{m} \in \operatorname{Max}(R)}(\underline{m} M)$ and by above $\bigcap_{\underline{m} \in \operatorname{Max}(R)} \underline{m} M=J(R) M$. Hence $\operatorname{Rad} M=J(\bar{R}) M$.

Lemma 2.12. Let $R$ be a ring and $M$ a multiplication $R$-module. Let $I M$ be an arbitrary non-zero proper submodule of $M$ for some ideal $I$ of $R$. Then $\operatorname{rad}(I M)=$ $(\operatorname{rad} I) M$ and $(\operatorname{rad}(I M): M)=\operatorname{radI}$, where $\operatorname{rad} I=r(I)$.

Proof. It is easy to show that $\operatorname{rad}(I M)=\bigcap_{p \in v(I)}(p M)$ (we recall that $v(I)=$ $\{p \in \operatorname{Spec}(R) \mid I \subseteq p\})$. By Lemma 2.11, $\operatorname{rad}(I M)=(\operatorname{rad} I) M$ and consequently $(\operatorname{rad}(I M): M)=\operatorname{rad} I$. 
We recall the following definition from [6].

Definition 2.13. A proper submodule $N$ of an $R$-module $M$ is said to be semiprime in $M$, if for every ideal $I$ of $R$ and every submodule $K$ of $M, I^{2} K \subseteq N$ implies that $I K \subseteq N$. Note that since the ring $R$ is an $R$-module by itself, a proper ideal $I$ of $R$ is semiprime if for every ideals $J$ and $K$ of $R, J^{2} K \subseteq I$ implies that $J K \subseteq I$.

Definition 2.14. There exists another definition of semiprime submodules in [4] as follows:

A proper submodule $N$ of the $R$-module $M$ is semiprime if whenever $r^{k} m \in N$ for some $r \in R, m \in M$ and positive integer $k$, then $r m \in N$.

By [7, Remark 2.6], we see that this definition is equivalent to Definition 2.13.

Definition 2.15. Let $M$ be an $R$-module and $N \leq M$. The envelope of the submodule $N$ is denoted by $E_{M}(N)$ or simply by $E(N)$ and is defined as $E(N)=\{x \in$ $M \mid \exists r \in R, a \in M ; x=r a$ and $r^{n} a \in N$ for some positive integer $\left.n\right\}$.

The envelope of a submodule is not a submodule in general.

Let $M$ be an $R$-module and $N \leq M$. If there exists a semiprime submodule of $M$ which contains $N$, then the intersection of all semiprime submodules containing $N$ is called semi-radical of $N$ and is denoted by $S-\operatorname{rad}_{M} N$, or simply $S-\operatorname{radN}$. If there is no semiprime submodule containing $N$, then we define $S-\operatorname{radN}=M$, in particular $S-\operatorname{rad} M=M$.

We say that $M$ satisfies the radical formula, or $M$ (s.t.r.f) if for every $N \leq M$, $\operatorname{radN}=\langle E(N)\rangle$. Also we say that $M$ satisfies the semi-radical formula, or $M$ (s.t.s.r.f) if for every $N \leq M, S-\operatorname{rad} N=\langle E(N)\rangle$. Now let $x \in E(N)$ and $P$ be a semiprime submodule of $M$ containing $N$. Then $x=r a$ for some $r \in R, a \in M$ and for some positive integer $n, r^{n} a \in N$. But $r^{n} a \in P$ and since $P$ is semiprime we have $r a \in P$. Hence $E(N) \subseteq P$. We see that $E(N) \subseteq \bigcap P$ (P is a semiprime submodule containing $N)$. So $\langle E(N)\rangle \subseteq S-\operatorname{rad} N$. On the other hand, since every prime submodule of $M$ is clearly semiprime, we have $S-\operatorname{rad} N \subseteq \operatorname{rad} N$. We conclude that $\langle E(N)\rangle \subseteq S-\operatorname{rad} N \subseteq \operatorname{radN}$ and as a result if $M$ (s.t.r.f) then it is also (s.t.s.r.f).

Lemma 2.16. Let $R$ be a ring and let $M$ be a multiplication $R$-module. Then every proper submodule of $M$ is a radical submodule, i.e., $\operatorname{rad} N=N$.

Proof. By [3, Theorem 2.12], $\operatorname{rad} N=\operatorname{rad}(N: M) M$. But $\operatorname{rad}(N: M) M \subseteq$ $\langle E(N)\rangle \subseteq \operatorname{rad} N$, hence $M$ (s.t.r.f) and so (s.t.s.r.f). Then $\langle E(N)\rangle=S-\operatorname{rad} N=$ $\operatorname{rad} N$ for every proper submodule $N$ of $M$. But by [6, Proposition 4.1], $S-\operatorname{rad} N=$ $N$ and therefore $\operatorname{radN}=N$. 
Corollary 2.17. Let $R$ and $M$ and $I M$ be as in Lemma 2.12. Then $I M=$ $(\operatorname{rad} I) M$.

Proof. Let $M$ be a multiplication $R$-module and $I M$ be an arbitrary non-zero proper submodule of $M$ for some ideal $I$ of $R$. By Lemma 2.12, $\operatorname{rad}(\operatorname{IM})=$ $(\operatorname{rad} I) M$ and by Lemma 2.16, $\operatorname{rad}(I M)=I M$. Therefore $I M=(\operatorname{rad} I) M$.

Theorem 2.18. Let $R$ be a ring and let $M$ be a multiplication $R$-module. Then $N$ is a primary submodule of $M$ if and only if it is a prime submodule of $M$.

Proof. $\Longleftarrow$. It is clear.

$\Longrightarrow$. Let $M$ be a multiplication $R$-module and let $N$ be an arbitrary primary submodule of $M$. Then by [2, Corollary 2], there exists a primary ideal $q(\operatorname{rad} q=p)$ of $R$ such that $N=q M$.

But by Lemma 2.16 and Corollary $2.17, q M=(\operatorname{rad} q) M=p M$. Therefore the proof is now completed.

Corollary 2.19. Let $R$ be a ring which satisfies ascending chain condition on semiprime ideals and let $M$ be a multiplication R-module. Then $M$ is a Noetherian $R$-module.

Proof. Let $M$ be a multiplication $R$-module. Then $M$ (s.t.r.f) and hence (s.t.s.r.f). Thus by [6, Proposition 4.1], every proper submodule of $M$ is a semiprime submodule of $M$. Now, let $I_{1} M \subseteq I_{2} M \subseteq I_{3} M \subseteq \ldots$ where $I_{i}$ are ideals of $R$ be ascending chain of submodules of $M$. Then $\left(I_{1} M: M\right) \subseteq\left(I_{2} M: M\right) \subseteq\left(I_{3} M: M\right) \subseteq \ldots$. But by [6, Proposition 2.3(ii)], $(N: M)$ is a semiprime ideal of $R$ for any semiprime submodule $N$ of $M$, hence by assumption there exists $n \in \mathbb{N}$ such that $\left(I_{n} M\right.$ : $M)=\left(I_{n+k} M: M\right)$ for each $k \in \mathbb{N}$. But then $\left(I_{n} M: M\right) M=\left(I_{n+k} M: M\right) M$ and so $I_{n} M=I_{n+k} M$. Therefore $M$ is a Noetherian $R$-module.

It should be noted that the above results (Lemma 2.16, Corollary 2.17, Theorem 2.18, Corollary 2.19) they are not necessarily true if $M=R$, the ring itself. Because according to $[6$, Theorem 4.4], $R$ (s.t.s.r.f) if we have one of the following.

(i) For every free $R$-module $F, F$ (s.t.s.r.f).

(ii) For every faithful $R$-module $B, B$ (s.t.s.r.f).

Acknowledgement. The authors would like to thank the referee for his/her careful reading and very useful comments and suggestions which improved the final version of this paper.

\section{REFERENCES}

[1] Barnard, A., "Multiplication modules", J. Algebra, 71:1(1981), 174-178. 
[2] Ebrahimi Atani, S., and Callialp, F., and Tekir, $\ddot{U}$., "A short note on the primary submodules of multiplication modules", Int. J. Algebra,1:8(2007), 381-384.

[3] EL-Bast, Z.A., and Smith, P.F., "Multiplication modules", Comm. Algebra, 16:4(1988), 755-779.

[4] Jenkins, J., and Smith, P.F., "On the prime radical of module over a commutative ring", Comm. Algebra, 20:12 (1992), 3593-3602.

[5] Lu, C.P., "Prime submodules of modules", Comment. Math. Univ. Sancti Pauli, 33:1(1984), 61-96.

[6] Tavallaee, H.A., "Modules satisfying the semi-radical formula", Hadronic Journal, 32(2009), 407-424.

[7] Tavallaee, H.A. and Ghalandarzadeh, S., "Semiprime submodules and envelope of modules", IUST-International Journal of Engineering Sciences, 14:4(2003), 131-142. 